\title{
Tendências de inovação em serviços de bibliotecas universitárias: estudo de caso do CRAI Universitat Pompeu Fabra em Barcelona, Espanha
}

\author{
Elisa Cristina Delfini Corrêa \\ Doutora; Universidade do Estado de Santa Catarina, Florianópolis, SC, Brasil \\ elisacorrea61@gmail.com \\ Miguel Ángel Marzal Garcia-Quismondo \\ Doutor; Universidade Carlos III, Madri, Espanha \\ mmarzal@bibuc3m.es
}

\begin{abstract}
Resumo: Neste artigo, são analisados produtos e serviços de base digital oferecidos pelo Centro de Recursos para Aprendizagem e Investigação da Universitat Pompeu Fabra em Barcelona, Espanha. O perfil da pesquisa é comparativo, bibliográfico e documental, e os dados foram coletados a partir de leitura e análise dos relatórios do New Media Consortium - NMC Horizon Report: 2015 Library Edition e NMC Horizon Report: 2017 Library Edition e da página web do Centro de Recursos para Aprendizagem e Investigação da Universitat Pompeu Fabra. Dados foram coletados também por meio de observação não participante realizada em visita à biblioteca do Campus del Poblenau, o que permite caracterizar a pesquisa como estudo de caso. Os dados foram submetidos a análise de conteúdo e foram categorizados. Os resultados demonstram a presença de muitas das tendências apresentadas nos relatórios do New Media Consortium, em especial aquelas de uso de tecnologia mais básica. É possível também observar, um pouco mais timidamente, a personalização de alguns serviços de acordo com as necessidades dos pesquisadores, assim como a oferta de ferramentas 2.0, bem como a entrega de conteúdos via dispositivos eletrônicos. O uso de tecnologias mais avançadas como Big Data, inteligência artificial ou tecnologia de localização, por exemplo, ainda não é encontrado. Conclui-se que são perceptíveis esforços para uma constante implementação de serviços e produtos de base digital, e, embora avanços tecnológicos mais sofisticados ainda não sejam encontrados, é possível identificar uma aproximação com as tendências apontadas pelos relatórios analisados.
\end{abstract}

Palavras-chave: Inovação. Bibliotecas universitárias. CRAI. Universitat Pompeu Fabra.

\section{Introdução}

A atualização digital pode se apresentar como uma questão de sobrevivência em muitas áreas de atuação nos dias atuais. As universidades estão atentas a esse 
fato, utilizando novas tecnologias digitais em suas diferentes atividades e processos administrativos e, em especial, promovendo estudos sobre o tema em seus cursos de graduação e pós-graduação.

Por conta disso surgiram novos tipos de bibliotecas, como as híbridas e as digitais ${ }^{1}$, nas quais a informação criada e distribuída eletronicamente recebe atenção especial. O desenvolvimento tecnológico e a evolução da internet impulsionaram mudanças radicais tanto nos processos de comunicação e divulgação da ciência quanto na própria maneira como esta se constrói atualmente, sendo os periódicos eletrônicos de acesso aberto e o movimento da ciência colaborativa alguns dos exemplos de transformações ocorridas na área científica.

Assim, as bibliotecas universitárias (BUs) representam ambientes nos quais a oferta de informação eletrônica se torna prioritária e nos quais são envidados maiores esforços para a criação e gestão de produtos e serviços de base digital. Ali as atividades de ensino, extensão e investigação científica de pesquisadores, docentes e alunos das universidades encontram o apoio necessário para o seu desenvolvimento acadêmico.

Sob essa perspectiva, este artigo se propõe a investigar tendências de uso dos espaços e recursos humanos e informacionais das BUs, bem como as possibilidades tecnológicas e digitais em seus serviços tendo como foco de análise os Centros de Recursos para Aprendizagem e Investigação (CRAIs), ambientes nos quais se transformaram muitas das bibliotecas universitárias da Espanha a partir dos anos 2000. Como objeto deste estudo de caso, será analisado o CRAI da Universitat Pompeu Fabra (UPF), em Barcelona, considerado um dos exemplos de sucesso da implantação desse modelo na Espanha, pois

A criação de uma estrutura poderosa para apoiar todas as áreas de atividade da universidade (ensino e aprendizagem e pesquisa) coloca a Universitat Pompeu Fabra em uma situação inovadora, com vantagem competitiva e referência em sua esfera de influência. (CABO RIGOL, 2006, p. 142, tradução nossa).

Os CRAIs espanhóis foram idealizados no final da década de 1990 a partir da criação da Red de Bibliotecas Universitarias (REBIUN), com base em 
modelos já existentes em outros países da Europa, como Inglaterra e Itália (GAVILÁN, 2008). A REBIUN inicia, em 2003, o processo de transformação das bibliotecas universitárias espanholas para implementação dos CRAIs.

A presente análise baseia-se na participação do CRAI no desenvolvimento da vida acadêmica, científica e universitária, com foco no contexto da informação digital, e tendo como parâmetro as tendências tecnológicas apresentadas nos relatórios do New Media Consortium (NMC): NMC Horizon Report: 2015 Library Edition e NMC Horizon Report: 2017 Library Edition. Buscou-se conhecer que produtos e serviços inovadores o CRAI UPF oferece à comunidade acadêmica, tendo como parâmetros as tendências para as BUs apresentadas nos relatórios do New Media Consortium, analisando o papel da biblioteca universitária no desenvolvimento da competência digital de seus interagentes ${ }^{2}$.

A pesquisa caracteriza-se como estudo de caso com coleta de dados bibliográfica (na qual a pesquisa se baseia teoricamente) e documental com observação não participante. A análise documental realizou-se por meio de pesquisa exploratória e descritiva da página web do CRAI da UPF (POMPEU FABRA, https.upf.edu a fim de descrever produtos e serviços inovadores e de base digital. Além disso, realizou-se pesquisa documental exploratória dos relatórios NMC Horizon: Library Edition (EDUCASE, 2019) referente a 2015 e 2017 a fim de proceder análise comparativa das tendências apresentadas nesse referencial e os serviços e produtos do CRAI UPF.

Por meio de análise de conteúdo, foram escolhidas categorias de tendências e foi identificado em quais pontos o caso estudado se aproxima das possibilidades previstas nos documentos do NMC para as bibliotecas universitárias a curto, médio e longo prazo. Por fim, realizou-se visita à biblioteca do Campus del Poblenou, para observação dos espaços onde os serviços e produtos podem ser acessados, completando assim a metodologia de estudo de caso. 


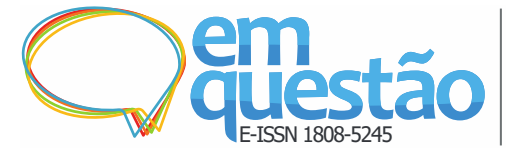

Tendências de inovação em serviços de bibliotecas universitárias: estudo de caso do CRAI Universitat Pompeu Fabra em Barcelona, Espanha

Elisa Cristina Delfini Corrrêa, Miguel Ángel Marzal Garcia-Quismondo

\section{Referencial teórico}

Para uma melhor compreensão da proposta desta pesquisa, faz-se necessário apresentar uma base teórica a respeito da proposta de implementação do modelo CRAI espanhol, bem como da premente necessidade da introdução de produtos e serviços de base digital em BU.

\subsection{Conhecendo o modelo CRAI}

Os Centros de Recursos para Aprendizagem e Investigação foram planejados para atuar como espaços alternativos e dinâmicos para a aprendizagem em um novo modelo educacional proposto pela União Europeia ainda na década de 1990. Esse modelo, adequado às demandas da sociedade da informação, tem como cenário as mudanças sociais decorrentes da popularização da internet enquanto plataforma educativa e em especial, a chamada Web social, ou Web 2.0. Seu desenho, além de incorporar recursos digitais, baseia-se em uma educação competencial, firmada na aquisição inicial de habilidades e destrezas com a finalidade da aquisição de capacidades e competências dentro de cada projeto de formação universitária (GARCÍA-QUISMONDO, 2008). Os CRAIs oferecem um espaço onde estão centralizados recursos e ferramentas para dar suporte ao modelo proposto pela União Europeia.

Gavilán (2008) lembra que os CRAIs não são uma criação espanhola e que centros de recursos como esses já existiam em outros países como Inglaterra e Itália. García-Quismondo (2008) aponta os Learning Resources Centres (LRC) em funcionamento nas universidades norte-americanas e inglesas como as principais referências estrangeiras para a transformação das BU em CRAI na Espanha.

Para García-Quismondo (2008) o CRAI representa uma excelente oportunidade para uma ascensão da biblioteca universitária não apenas porque ele faz crescer a qualidade e a excelência de seus serviços, mas em especial porque ressalta a biblioteca universitária enquanto elemento essencial no novo processo educacional demandado pela sociedade do conhecimento.

Os CRAIs têm as seguintes características: 
a) são centrados no sujeito, não no objeto;

b) são espaços flexíveis e atrativos de socialização do conhecimento;

c) têm como missão facilitar o acesso e difusão dos recursos de informação e colaborar nos processos de criação do conhecimento;

d) baseiam-se na convergência de suportes físicos e digitais, para oferecer produtos e serviços com recursos materiais e humanos e recursos de informação e aprendizagem integrados à web;

Além disso, os CRAI têm por finalidade:

a) atuar como portal de informação para um repositório digital de documentos acessíveis em rede;

b) dar suporte a todas as atividades educacionais no ambiente universitário, oferecendo infraestrutura tecnológica e equipamentos;

c) integrar em um único espaço serviços já existentes, mas dispersos ou duplicados na universidade;

d) contribuir para a inovação no trabalho docente e para a acessibilidade a fontes e recursos de informação a toda a comunidade acadêmica, dando melhor assistência a professores, alunos e técnicos;

e) reduzir tempo e burocracia, melhorando o aproveitamento dos recursos de informação na universidade;

f) permitir o compartilhamento e a colaboração característicos da web 2.0, potencializando a comunicação entre os diferentes atores da universidade, como bibliotecários e professores; com seus resultados positivos, aumentar a visibilidade e a reputação da universidade como um todo;

Em relação às vantagens que os CRAIs podem trazer às BU, GarcíaQuismondo (2008) afirma que a transformação das bibliotecas em centros de recursos torna sua presença mais efetiva no processo educacional pois, apesar da introdução das tecnologias de informação e comunicação (TIC) no ambiente 
universitário como um todo, as Bus ainda mantinham uma atuação periférica nas atividades de ensino, pesquisa e extensão.

A partir de sua transformação em CRAI, é possível concretizar o que este autor chama de quatro estímulos "revolucionários": (1) caracterização dos conteúdos digitais educativos (em suas características de granularidade, reutilização, dinamicidade, interatividade e virtualidade: objetos de aprendizagem digital); (2) aprendizagem colaborativa; (3) web 2.0; (4) modelo educativo competencial. Essas transformações sociais motivadas pelo desenvolvimento tecnológico reúnem o conjunto de características que fundamentam o conceito dos CRAIs evidenciando os papéis baseados em tecnologias e na formação de competências para o uso e geração de informação digital.

Ainda segundo García-Quismondo (2008) o CRAI representa uma oportunidade ímpar para a potencialização das atividades das Bus e dos bibliotecários, favorecendo uma aproximação muito maior destes com a comunidade acadêmica e a participação da própria universidade na sociedade do conhecimento.

Gavilán (2008) aponta para o papel fundamental das BU em sua atuação enquanto CRAI afirmando que, nesse novo contexto, o trabalho bibliotecário deverá constituir um dos indicadores para medir a qualidade da própria universidade. Esse mesmo autor também ressalta que a conversão das BU em CRAIs permitirá a criação de diferentes serviços, quais sejam: serviço de informação global de acolhida na universidade; serviço de biblioteca; serviço informático para os estudantes; laboratório de idiomas; busca ativa de emprego; sala de estudos; suporte para a formação do professor; criação e elaboração de materiais docentes e multimídia; apresentações e debates; reprografia e outras facilidades diretas para o interagente. Para Gavilán (2008)

Neste contexto, a biblioteca se converte em uma sala de autoformação, onde são elaborados produtos e objetos de aprendizagem de acordo com o novo modelo de educação [proposto pela União Europeia]. (GAVILÁN, 2008, p.7, tradução nossa) 
Gavilán (2008) ainda afirma que a transformação das BUs em CRAIs permite integrar serviços ligados a projetos educativos relativos à informação e tecnologia para professores e técnicos; transformar a biblioteca universitária de "instituição de apoio" em um centro ativo de aprendizagem mais próximo à docência e à pesquisa, um ambiente híbrido orientado à investigação e ao aprendizado, integrado a serviços já existentes na universidade e, ainda, conforme esse autor:

a) integrar/conhecer a missão da universidade, seu potencial tecnológico, seu repositório institucional e suas publicações;

b) integrar/conhecer os serviços da biblioteca, a estrutura física e de recursos humanos disponíveis/possíveis, os horários de atendimento, os equipamentos convergentes e acessíveis;

c) oferecer serviços de apoio à produção docente (espaço, orientação, recursos informacionais e tecnológicos);

d) atuar como centro de recursos educativos básicos para a comunidade universitária, contribuindo para uma reestruturação da organização universitária.

Castro Filho (2008) aponta as mesmas características destacadas pelos autores mencionados e ressalta que o modelo CRAI é centrado no sujeito e não no objeto e que, por isso, as BUs transformam-se em espaços atrativos e de socialização.

\subsection{Bibliotecas universitárias e as tecnologias de informação e comunicação}

Não é novidade que as TICs levaram bibliotecas, arquivos e museus a promoverem muitas transformações em sua forma de organização, de criação de produtos e de prestação de serviços. No ambiente das BUs, essas transformações são percebidas de maneira bastante contundente. Segundo Moreira (2018)

A percepção de que há uma reciprocidade entre as BU e as novas tecnologias de informação e comunicação está clara quando percebemos que, para consolidar a modernização das bibliotecas, as tecnologias digitais são essenciais. (MOREIRA, 2018, p.98) 
No campo da educação superior essa necessidade é premente e muitas são as possibilidades que se apresentam às BUs. Anglada (2012) afirma que "As bibliotecas universitárias, com maior ou menor consciência da profundidade da mudança, estiveram no passado - e ainda estão no presente - na vanguarda da aplicação de inovações tecnológicas aos seus serviços." (ANGLADA, 2012, p. 554, tradução nossa). Dentre as possibilidades, o autor destaca repositórios e acesso aberto, renovação de espaços, serviços para pesquisadores, instrumentos de descoberta e livros eletrônicos (ANGLADA, 2012).

Castro Filho e Vergueiro (2011) analisam que

\begin{abstract}
Novas propostas de ensino-aprendizagem surgiram com o advento das Tecnologias de Informação e Comunicação. As bibliotecas universitárias devem estar inseridas nesse processo, uma vez que sua missão é facilitar o acesso e a difusão dos recursos de informação e colaborar nos processos de criação do conhecimento, a fim de viabilizar os objetivos da Universidade. (CASTRO FILHO; VERGUEIRO, 2011, p. 32).
\end{abstract}

Assim, embora seja patente a dificuldade em acompanhar inovações tecnológicas com a rapidez necessária e embora as bibliotecas universitárias caminhem a passos lentos nesse quesito, são notórias as mudanças verificadas nesses ambientes. Na medida do possível, essas bibliotecas buscam acompanhar o desenvolvimento de tecnologias a serviço da pesquisa científica e torna-se cada vez mais comum a oferta de serviços digitais à comunidade universitária.

Zaninelli, Nogueira e Peres (2019) apontam que

Apesar da finalidade das BUs permanecer a mesma com o passar dos anos, os processos utilizados no oferecimento de serviços e produtos mudaram, visto que as transformações tecnológicas causaram alterações em tais unidade. (ZANINELLI; NOGUEIRA; PERES, 2019, p.12)

Silveira (2014) identifica áreas nas quais as tecnologias apresentam interferência nas BUs, como

$\{\ldots\}$ o aumento do fluxo de informações, a fluidez das relações interpessoais, a automatização de diversos processos e produtos, a quebra de paradigmas e o surgimento de novos conceitos como a globalização e as tecnologias da informação. (SILVEIRA, 2014, p. 73) 
Dessa maneira, as BUs mantêm suas raízes no que tange a seus objetivos de apoio e facilitação da geração e da disseminação do conhecimento científico nas universidades; contudo, a partir do uso de tecnologias, agregam valor a muitos de seus serviços e produtos inovadores e potencializam seu alcance:

[...] a inovação é vista como um aspecto que agrega valor para o serviço ou bem de uma determinada organização e tem recebido a atenção dos estudiosos devido às mudanças que proporcionaram aos clientes/usuários dos serviços, muitos influenciados pelo impacto que as tecnologias ocasionaram e pelas mudanças que decorreram deste aspecto. (BAREGHEH; ROWLEY; SAMBROOK, $2009^{3}$ apud NEVES, 2018, p.47).

No entanto, são muitos os desafios a serem vencidos para que as BUs possam modernizar seus serviços e produtos e adaptá-los à era digital. Dentre esses desafios, é extremamente necessário munir-se de informações não apenas a respeito de sua comunidade acadêmica, mas, igualmente, a respeito das tendências tecnológicas aplicáveis às bibliotecas universitárias, a fim de adaptar essas tendências ao próprio contexto. Para tal, os relatórios New Media Consortium Horizon apresentam-se como uma ferramenta bastante útil, como abordado a seguir.

\section{Tendências e possibilidades para bibliotecas universitárias no contexto digital}

Conhecer o que está acontecendo em bibliotecas acadêmicas e de pesquisa mundo afora, no que tange à implementação de recursos digitais, é uma das maneiras mais eficazes para acompanhar esse movimento de modernização tecnológica. Organizações como o New Media Consortium Horizon Project, iniciativa mundial de pesquisa sobre tendências e desafios tecnológicos no campo da educação, podem ser muito úteis aos gestores de bibliotecas interessados em operar mudanças em seus ambientes informacionais.

O NMC Horizon Report: Library Edition é um relatório bianual de pesquisa sobre tendências e possibilidades digitais para bibliotecas acadêmicas e de pesquisa. Durante o período de coleta de dados, compreendido entre setembro e novembro de 2018, estavam disponíveis, na página do NMC, os 
relatórios de 2004 a 2017, que descrevem possíveis mudanças de longo, médio e curto impacto e que remetem ao que há de mais recente em bibliotecas em todo o mundo. Apresentam tendências e novidades, citam exemplos concretos de aplicação e discutem aspectos políticos e de gestão que influenciam a implementação dessas novidades.

Os relatórios têm como objetivo ser uma referência e um guia de planejamento tecnológico para bibliotecários, gerentes e funcionários de bibliotecas, formuladores de políticas e tecnólogos. Os especialistas de diferentes instituições que colaboram na publicação do relatório buscam apresentar um cenário tecnológico no prazo de cinco anos futuros, discutindo as tendências que impulsionam as bibliotecas acadêmicas e as bibliotecas de pesquisa e os desafios que elas possivelmente terão de enfrentar para implementar soluções tecnológicas para sua comunidade alvo.

Os estudos orientam-se por dez afirmações-base, que ressaltam o compromisso das bibliotecas com o seu papel social no contexto tecnológico universitário. Dessas afirmações, interessa mais diretamente a afirmação seis que preconiza que estender a fluência digital é responsabilidade central. A partir dessa ideia, fica patente a responsabilidade assumida pelas bibliotecas pela competência digital de sua comunidade de interagentes e a necessidade de manter-se sempre em dia com as novidades tecnológicas. As bibliotecas devem, portanto, posicionar-se para

[...] liderar os esforços que desenvolvem a maturidade digital dos usuários, garantindo que eles dominem o uso responsável e criativo das tecnologias, incluindo a identidade online, o protocolo de comunicação adequado e os direitos e responsabilidades. (BECKER et al., 2017, p.22. tradução nossa).

Fica clara a responsabilidade do bibliotecário atuante nesse ambiente informacional.

Os relatórios apresentam, primeiramente e de forma mais abrangente, as tendências mundiais na área de informação digital que podem acelerar as mudanças no ambiente das bibliotecas universitárias e de pesquisa. Essas tendências podem provocar 'impactos' nesses ambientes e são analisadas a partir de suas implicações a curto, médio e longo prazo. São apontadas as 
dificuldades que essas instituições enfrentam para implementar novos produtos e serviços em diferentes níveis, são descritos e discutidos os desenvolvimentos de tecnologia educativa para a educação superior, considerados os mais importantes do período coberto por cada relatório.

Para este trabalho, foram estudados os relatórios de 2015 e 2017 (elaborados em parceria com a EDUCAUSE Learning Initiative), com vistas a apresentar uma visão abrangente das inovações em bibliotecas universitárias, acadêmicas e de pesquisa. Com base na identificação dessas tendências foi analisado comparativamente o CRAI UPF, a partir da oferta de produtos e serviços de base tecnológica.

Abaixo estão descritas, de forma sintética, as tendências, possibilidades e prazos de implementação para as bibliotecas constantes nos relatórios analisados.

\subsection{Relatório NMC Horizon - Library Edition 2015}

As alíneas a seguir apresentam as categorias de prazos para a implementação das tendências tecnológicas visualizadas pelos especialistas do consórcio NMC/EDUCASE. A implementação de cada grupo de ferramentas está prevista em períodos de tempo que podem ser de um a cinco anos (ou mais), dependendo dos desafios e impactos que envolvem em sua negociação, planejamento e aplicação.

Em relação ao quesito impacto, são considerados de curto, médio e longo prazo:

a) tendências com impacto de longo prazo (cinco anos ou mais): aumentar a acessibilidade ao conteúdo de pesquisas, repensar o espaço da biblioteca e acompanhar a natureza dos registros acadêmicos;

b) tendências com impacto de médio prazo (três a cinco anos): aumentar o foco na gestão de dados de pesquisa;

c) tendências com impacto de curto prazo (um ou dois anos): desenvolver o valor da experiência do usuário (user experience UX) e priorizar conteúdo e entrega para celular. 
Os principais desafios a serem vencidos na implementação das tendências, são divididos em três categorias:

a) acessíveis ("nós os entendemos e sabemos como resolvê-los"): incluir as bibliotecas acadêmicas e de pesquisa nos currículos universitários, melhorar a competência digital;

b) difíceis ("nós os entendemos, mas eles requerem soluções difíceis de implementar"): competir com modos alternativos de descoberta, repensar o papel e habilidades do bibliotecário;

c) muito complexos (“difíceis de definir e mais ainda de resolver"): assumir a necessidade de uma mudança radical e gerenciar a obsolescência do conhecimento.

Das mudanças passíveis de serem implementadas em bibliotecas num prazo inferior a um ano, o relatório destaca: makerspaces e aprendizagem online; num prazo de dois a três anos: visualização da informação, web semântica e linked data; num prazo de quatro a cinco anos: inteligência de localização e máquinas inteligentes.

\subsection{Relatório NMC Horizon - Library Edition 2017}

Da mesma forma, o relatório de 2017 apresenta as seguintes análises:

a) tendências com impacto de longo prazo (cinco anos ou mais): colaborações entre instituições e acompanhamento do caráter evolutivo dos documentos acadêmicos;

b) tendências com impacto de médio prazo (três a cinco anos): entender os usuários como criadores e repensar espaço das bibliotecas;

c) tendências com impacto de curto prazo (um ou dois anos): desenvolver gestão de dados de pesquisa e valorizar a UX.

Os principais desafios a serem vencidos na implementação das tendências, são novamente divididos em três categorias:

a) acessíveis ("nós os entendemos e sabemos como resolvê-los"): melhorar a acessibilidade dos serviços e recursos das bibliotecas; desenvolver a competência digital; 


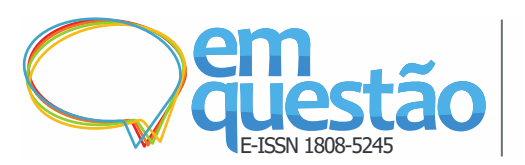

Tendências de inovação em serviços de bibliotecas universitárias: estudo de caso do CRAI Universitat Pompeu Fabra em Barcelona, Espanha

Elisa Cristina Delfini Corrrêa, Miguel Ángel Marzal Garcia-Quismondo

b) difíceis ("nós os entendemos, mas eles requerem soluções difíceis de implementar"): adaptar desenhos organizacionais ao futuro do trabalho; desenvolver a manutenção de projetos de integração contínua, interoperabilidade e colaboração;

c) muito complexos (“difíceis de definir e mais ainda de resolver"): vencer pressões políticas e econômicas; assumir a necessidade de enfrentar mudanças radicais.

O relatório identificou que as tendências possíveis e seus prazos de implementação em bibliotecas acadêmicas e de pesquisa eram: em prazo inferior a um ano, big data e tecnologias para atividades acadêmicas digitais; num prazo de dois a três anos, identidade em linha e plataformas de serviços bibliotecários, e num prazo de quatro a cinco anos, inteligência artificial e internet das coisas.

Para a análise do CRAI UPF, as categorias e tendências apontadas nos dois relatórios acima descritos são as seguintes:

a) makerspaces: talvez uma das tendências mais aceitas nas bibliotecas atualmente, são espaços que permitem a manipulação e empréstimo de objetos para geração de conhecimentos práticos e ideias inovadoras;

b) aprendizagem virtual: a biblioteca pode promover oportunidades de aprendizagem virtual oferecendo cursos e tutoriais com metodologias e ferramentas específicas em parceria com cursos da universidade, integrando seus serviços aos planos de ensino. A biblioteca pode estimular a produção multimídia, selecionar conteúdos digitais e criar recursos didáticos tecnológicos. Muitas bibliotecas promovem cursos presenciais e a distância sobre competência em informação e produzem ativos digitais para acessibilidade e melhora de aprendizagem;

c) visualização da informação: as bibliotecas podem contribuir para a distribuição de informação técnica e/ou científica com formatos como os infográficos, cuja visualização permite que permitem uma melhor compreensão dos dados divulgados. Pode-se utilizar de metodologias como o design thinking e fazer uso de software 
criativoPode utilizar-se de metodologias como o design thinking e fazer uso de software criativo;

d) web semântica e linked data: essas tendências parecem já fazer parte da rotina de muitas bibliotecas universitárias e de pesquisa, dada a importância para a área científica. A utilização da web semântica e ferramentas para descoberta e enlace de dados podem ser identificadas em boa parte dessas bibliotecas;

e) inteligência de localização: a utilização de recursos inteligentes de localização permitirá gerar conteúdo de acordo com a localização do usuário, bem como oferecer informações específicas e personalizadas a partir de sua posição no ambiente da biblioteca, inclusive com tecnologia 3D;

f) máquinas inteligentes: essa tecnologia permite filtrar grandes quantidades de dados e também aperfeiçoar autosserviços adequados a partir da interação com os usuários;

g) big data: esse recurso serve também para uma personalização de serviços, embora o relatório mencione possibilidades mais polêmicas como a liberação de dados para a universidade ou o governo;

h) tecnologias para atividades acadêmicas digitais: trata-se da implementação de um conjunto de ferramentas digitais e computacionais atualmente sendo usado para promover a oferta de tecnologia para suportar o acesso, recuperação e aplicação de conhecimentos. Esses produtos incluem mídia digital, websites, arquivos de informação acadêmica e exposições digitais;

i) plataformas de serviços bibliotecários: essas plataformas representam uma nova abordagem conceitual para a biblioteca, atendendo às necessidades atuais de um sistema centrado na web. Elas permitem gerir recursos impressos e eletrônicos e mantêm o ritmo das necessidades operacionais de mudança nos ambientes de biblioteca; em essência, oferecem às bibliotecas oportunidades de abordar conscientemente os meandros de conteúdo e dos fluxos de 
trabalho e de descoberta para uma melhor integração com a comunidade acadêmica;

j) identidade online: também chamada de identidade digital, parte da ideia de que tudo o que interage no campo digital possui ou adquire um conjunto de dados ou atributos, inerentes e variáveis, que identificam unicamente uma pessoa ou entidade on-line. Para as bibliotecas, esse conceito é importante na medida em que amplia a visão sobre competência digital e sobre a atribuição de autor da rede, o que permite acompanhar o crescimento da produção de um autor e especificar relações de conteúdo com outras pesquisas;

k) inteligência artificial: este conceito é bem conhecido e tem relação com as máquinas inteligentes;

1) internet das coisas: consiste em objetos equipados com poder de computação através de processadores integrados ou sensores capazes de transmitir informações entre redes. Seu uso está sendo estudado por gestores de bibliotecas, pois pode auxiliar em sua sustentabilidade em termos de economia de energia e de conectividade de objetos a recursos que possam contextualizar seu uso, aproveitar dados vinculados e conectar a biblioteca a recursos informacionais em nuvem.

\section{Estudo de caso do CRAI UPF - Barcelona}

O modelo CRAI implementado na UPF tem como base a integração de setores diversos da universidade em trabalho conjunto, porém, com serviços prestados em diferentes espaços nos campi. Os serviços, desenvolvidos por equipes multidisciplinares, das quais participam bibliotecários, cientistas da computação e técnicos audiovisuais, têm como meta distribuir informação e promover a geração de conhecimentos por meio do ensino e pesquisa.

Foi necessário percorrer um extenso caminho de mudanças internas e adaptações a fim de implementar o novo modelo organizacional iniciado em 2003, bem como empreender um forte trabalho de conscientização acerca das vantagens e benefícios da nova proposta, não apenas do corpo técnico da 
universidade, mas de toda a comunidade universitária. Desde o seu início, o CRAI UPF busca exercer suas atividades de maneira estrategicamente planejada, divulgando periodicamente relatórios que atestem à sociedade seu desenvolvimento e o valor científico e social de sua proposta. O planejamento estratégico é realizado anualmente na Gestão por Objetivos (DPO), que estabelece quais objetivos operacionais devem ser realizados para a estratégia definida (UPF, 2005).

O relatório de gestão que cobre os anos 2016 e 2017 destaca as principais conquistas do período, cujos impactos se refletem no apoio prestado pela biblioteca à aprendizagem, ao ensino e à gestão universitários. Dentre as conquistas são mencionadas a criação do Portal de Pesquisa das Universidades da Catalunha, o empréstimo de notebooks e o serviço de gerenciamento de dados de pesquisa (GUIES BBTIC, [2019]). Para atingir esses objetivos, o CRAI UPF atua especialmente em cooperação com o Departamento de Informática dessa universidade.

Dado o interesse desta pesquisa, foram analisadas as informações disponibilizadas na página web do CRAI UFP - Biblioteca e Informática. Para a análise dos serviços com base digital, os dados para a pesquisa foram coletados a partir do link "Serviços e Recursos” e, dentro deste, "Todos os serviços de AZ”.

A utilização de tecnologias pelo CRAI UPF é evidente em uma gama de serviços prestados pelas suas bibliotecas, abaixo relacionados e descritos. A partir de uma comparação deles com as tendências apresentadas nos relatórios da NMC Horizon, é possível analisar o desenvolvimento do CRAI em questão em função das possibilidades tecnológicas da sociedade contemporânea. Estão listados, além dos serviços, os espaços utilizados para apoio à aprendizagem com base digital; para a coleta desses dados, buscaram-se as informações do link "Espaços". Para observação dos espaços disponíveis no CRAI UPF Poblenou, procedeu-se a uma visita presencial no dia 11 de outubro de 2018, quando foi possível conhecer os laboratórios e salas tanto da biblioteca quanto do campus conhecido como "Factoria", uma vez que o local que abriga a UPF atualmente 
era uma antiga fábrica têxtil. Os dados coletados revelam os seguintes produtos e serviços oferecidos de base digital:

a) oferta e criação de serviços digitais:

- acesso Virtual Private Network (VPN): acesso a recursos do computador disponíveis apenas dentro da rede UPF;

- acesso aos recursos fora da UPF através dos Serviços de Acesso aos Recursos Eletrônicos (SARE);

- certificação Acreditação de Competências em Tecnologias de Informação e Comunicação (ACTIC): certificado de competência digital;

- assessoria tecnológica: suporte técnico, orientação e proposição de soluções customizadas e personalizadas;

- acesso à biblioteca digital com bases de dados, e-livros e erevistas;

- orientação para a criação de vídeos tutoriais;

- digitalização de materiais de ensino;

- utilização de disco de rede: compartilhamento de dados com grupos de pesquisa ou possibilidade de copiar dados de fora do seu escritório;

- utilização de microdados: suporte e gerenciamento;

- formação em competência digital: cursos para estudantes de graduação e pós-graduação;

- produção de massive open online courses (MOOCs): produção de curso on-line, aberto e massivo, sob a direção do Centro de Inovação em Aprendizagem e Conhecimento (sigla em inglês CLIK);

- orientação no planejamento e disseminação de dados de pesquisa;

- disponibilização de softwares para diferentes usos científicos e pessoais;

- supercomputação: pesquisa intensiva em termos de computação, diferentes infraestruturas para cálculos intensivos; 
- virtualização: máquinas virtuais que compartilham os recursos livres de CPU, memória, disco e conexão de rede;

b) produtos de base digital:

- repositório digital da UPF;

- repositórios de código: código-fonte, páginas da web ou documentação;

- Portal de Produção Científica (PPC);

- MyApps: plataforma em nuvem que permite acessar aplicativos e usá-los sem precisar instalá-los no seu dispositivo;

c) espaços:

- salas de aula de informática;

- cabines individuais e salas de conferência;

- estúdios e salas para edição de vídeos;

- HackLAB ${ }^{4}$

- laboratórios.

\section{Análise}

A comparação entre as mudanças possíveis e tendências apontadas pelo relatório NMC Horizon e a oferta de serviços, produtos e espaços demonstra que o CRAI UFP utiliza tecnologias mais simples e acessíveis para a solução de problemas de uso e gestão de informação científica. Serviços com base em tecnologias mais avançadas como inteligência artificial, máquinas inteligentes, internet das coisas ou mesmo big data e localização não foram identificados na coleta de dados.

Por outro lado, verificou-se que foram implementados alguns serviços que seguem as tendências previstas nos relatórios de 2015 e 2017; investiu-se na criação e disponibilização de serviços e ferramentas de gestão de dados de pesquisa, provendo os usuários de acesso, suporte e armazenamento. Um pouco mais timidamente, é possível também observar a personalização de alguns serviços de acordo com as necessidades dos pesquisadores, assim como a oferta de ferramentas 2.0 que permitem personalização de acordo com o objetivo de 
sua utilização e como a entrega de conteúdos via dispositivos eletrônicos como telefones celulares.

Espaços de criação, como o HackLAB e como os demais laboratórios da UPF, acompanham as tendências de repensar os espaços da biblioteca e de possibilitar que a comunidade acadêmica atue como criadora de conhecimentos e soluções para diferentes demandas, colocando à disposição equipamentos de informática e de produção de vídeo e som.

São visíveis os esforços de aumentar a acessibilidade ao conteúdo de pesquisas a partir da criação de bibliotecas digitais, portal e repositório institucional. O uso de web semântica, de plataformas e de softwares também está presente.

É possível identificar colaboração entre instituições nas parcerias para a oferta de cursos; também fica patente a colaboração dentro da própria UPF, em especial, com o departamento de informática. Essas parcerias resultam na criação de ferramentas e serviços tecnológicos que, embora de maneira ainda incipiente, abrem caminhos para que os pesquisadores acompanhem a evolução dos documentos acadêmicos em rede e participem desse caráter evolutivo, previsto nos Relatórios NMC.

Não foi identificado o uso de equipamentos ou de tecnologia avançada como drones, holografia ou realidade aumentada. Contudo, nos espaços utilizados pelo CRAI estão disponíveis impressoras 3D, e percebe-se a utilização de vários recursos de nuvem e internet.

É possível afirmar que, para a implementação desses serviços, alguns dos desafios levantados nos relatórios foram vencidos, como pode ser verificado na permanência de projetos de integração contínua e de colaboração. Merece destaque, para a presente pesquisa, a redefinição do papel do bibliotecário, especialmente em relação à sua atuação como educador no contexto digital nas estratégias de aprendizagem virtual. Paralelamente, a biblioteca avança na direção de cumprir o desafio de melhorar a competência digital da comunidade acadêmica. 
Tendências de inovação em serviços de bibliotecas universitárias: estudo de caso do CRAI Universitat Pompeu Fabra em Barcelona, Espanha

Elisa Cristina Delfini Corrrêa, Miguel Ángel Marzal Garcia-Quismondo

Quadro 1 - Análise comparativa entre serviços e espaços UPF e relatórios NMC Horizon

\begin{tabular}{|c|c|c|c|}
\hline $\begin{array}{l}\text { MUDANÇAS } \\
\text { VERIFICADAS }\end{array}$ & $\begin{array}{l}\text { TENDÊNCIAS } \\
\text { ATINGIDAS }\end{array}$ & $\begin{array}{l}\text { SERVIÇOS/ } \\
\text { ESPAÇOS }\end{array}$ & $\begin{array}{l}\text { DESAFIOS } \\
\text { VENCIDOS }\end{array}$ \\
\hline MAKERSPACES & $\begin{array}{lr}\begin{array}{l}\text { Repensar } \\
\text { espaço } \\
\text { biblioteca }\end{array} & \text { da } \\
& \\
\begin{array}{l}\text { Usuários } \\
\text { criadores }\end{array} & \text { como }\end{array}$ & $\begin{array}{l}\text { Estúdios, } \\
\text { HackLAB, } \\
\text { laboratórios, } \\
\text { salas de edição } \\
\text { de vídeo }\end{array}$ & $\begin{array}{l}\text { manutenção de projetos de } \\
\text { integração contínua }\end{array}$ \\
\hline $\begin{array}{l}\text { APRENDIZAGEM } \\
\text { VIRTUAL }\end{array}$ & $\begin{array}{l}\begin{array}{l}\text { Experiência do } \\
\text { usuário (UX) }\end{array} \\
\begin{array}{l}\text { Colaboração } \\
\text { entre instituições }\end{array} \\
\begin{array}{l}\text { Usuários como } \\
\text { criadores }\end{array}\end{array}$ & $\begin{array}{ll}\text { ACTIC, } & \\
\text { formação em } \\
\text { competência } \\
\text { digital, produção } \\
\text { de aulas MOOC, } \\
\text { produção de } \\
\text { vídeos, salas } & \text { de } \\
\text { aula } & \text { de } \\
\text { informática } & \end{array}$ & $\begin{array}{l}\text { melhorar a competência } \\
\text { digital } \\
\text { repensar o papel e } \\
\text { habilidades do bibliotecário } \\
\text { manutenção de projetos de } \\
\text { integração contínua, } \\
\text { interoperabilidade } \\
\text { colaboração }\end{array}$ \\
\hline $\begin{array}{l}\text { VISUALIZAÇÃO DA } \\
\text { INFORMAÇÃ̃O }\end{array}$ & $\begin{array}{l}\text { Usuários como } \\
\text { criadores }\end{array}$ & $\begin{array}{l}\text { Criação } \quad \mathrm{de} \\
\text { vídeos }\end{array}$ & \\
\hline $\begin{array}{l}\text { WEB } \\
\text { SEMÂNTICA/LINKED } \\
\text { DATA }\end{array}$ & $\begin{array}{l}\text { Aumento da } \\
\text { acessibilidade ao } \\
\text { conteúdo de } \\
\text { pesquisas, } \\
\text { acompanhamento } \\
\text { da natureza dos } \\
\text { registros } \\
\text { acadêmicos e } \\
\text { aumento do foco } \\
\text { na gestão de } \\
\text { dados de pesquisa } \\
\text { Caráter evolutivo } \\
\text { dos documentos } \\
\text { acadêmicos }\end{array}$ & 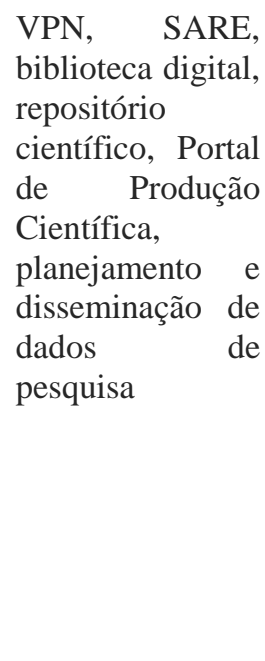 & $\begin{array}{l}\text { manutenção de projetos de } \\
\text { integração contínua, } \\
\text { interoperabilidade } \\
\text { colaboração }\end{array}$ \\
\hline $\begin{array}{l}\text { TECNOLOGIAS PARA } \\
\text { ATIVIDADES } \\
\text { ACADÊMICAS }\end{array}$ & $\begin{array}{ll}\text { Aumento } & \text { da } \\
\text { acessibilidade } & \text { ao } \\
\text { conteúdo } & \text { de } \\
\text { pesquisas, } & \\
\text { acompanhamento } \\
\text { da natureza } & \text { dos }\end{array}$ & $\begin{array}{l}\text { VPN, SARE, } \\
\text { microdados, } \\
\text { aplicativos } \\
\text { (Myapps), } \\
\text { softwares, } \\
\text { supercomputação }\end{array}$ & $\begin{array}{l}\text { manutenção de projetos de } \\
\text { integração contínua, } \\
\text { interoperabilidade } \\
\text { colaboração }\end{array}$ \\
\hline
\end{tabular}




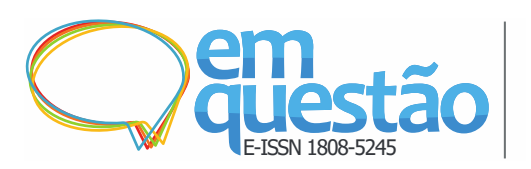

Tendências de inovação em serviços de bibliotecas universitárias: estudo de caso do CRAI Universitat Pompeu Fabra em Barcelona, Espanha

Elisa Cristina Delfini Corrrêa, Miguel Ángel Marzal Garcia-Quismondo

\begin{tabular}{|c|c|c|c|}
\hline & $\begin{array}{l}\text { registros } \\
\text { acadêmicos } \mathrm{e} \\
\text { aumento do foco } \\
\text { na gestão de } \\
\text { dados de pesquisa }\end{array}$ & , virtualização & \\
\hline $\begin{array}{l}\text { PLATAFORMAS DE } \\
\text { SERVIÇOS } \\
\text { BIBLIOTECÁRIOS }\end{array}$ & 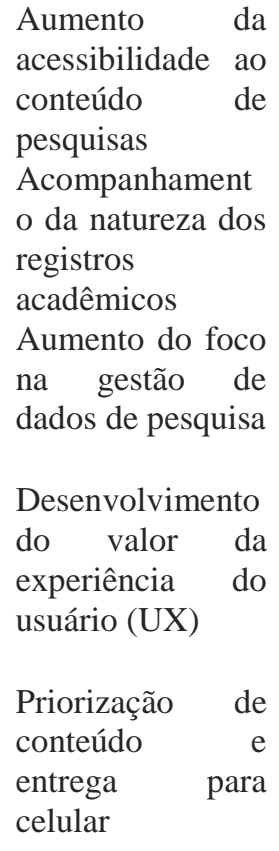 & $\begin{array}{l}\text { Assessoria } \\
\text { tecnológica, } \\
\text { disco de rede, } \\
\text { repositório } \\
\text { científico e de } \\
\text { códigos, } \\
\text { ferramentas 2.0, } \\
\text { supercomputação } \\
\text {, virtualização }\end{array}$ & $\begin{array}{l}\text { manutenção de projetos de } \\
\text { integração contínua, } \\
\text { interoperabilidade } \\
\text { colaboração }\end{array}$ \\
\hline IDENTIDADE ONLINE & $\begin{array}{lr}\begin{array}{l}\text { Experiência } \\
\text { usuário (UX) }\end{array} & \text { do } \\
\text { Priorização } & \text { de } \\
\text { conteúdo } & \text { e } \\
\text { entrega } & \text { para } \\
\text { celular } & \end{array}$ & $\begin{array}{l}\text { Assessoria } \\
\text { tecnológica, } \\
\text { ferramentas } 2.0\end{array}$ & $\begin{array}{l}\text { manutenção de projetos de } \\
\text { integração contínua, } \\
\text { interoperabilidade } \\
\text { colaboração }\end{array}$ \\
\hline
\end{tabular}

Fonte: Dados da pesquisa.

O quadro acima aponta para um cenário no qual boa parte das tendências apresentadas nos relatórios NMC Horizon Report: Library Edition 2015 e 2017 (BECKER et al., 2017; JOHNSON et al., 2015) foram desenvolvidas e implementadas. Os serviços e produtos desenvolvidos e ofertados representam, segundo os relatórios, aqueles passíveis de criação e implementação em prazos menores. Os serviços de maior complexidade, que necessitam de investimentos 
em tecnologias mais recentes, como máquinas inteligentes, big data e internet das coisas, não foram encontrados no CRAI.

No entanto, é preciso levar em consideração o fato de que os relatórios indicam um prazo de cinco anos para a implementação de atividades de maior complexidade, o que significa que, de acordo com as datas de publicação dos relatórios NMC analisados (2015 e 2017) e com o ano em que a pesquisa foi realizada, 2018, fica compreensível a ausência de produtos e serviços que demandam o uso de tecnologias avançadas.

\section{Considerações finais}

As bibliotecas universitárias fazem parte de todo um sistema de unidades de ensino, pesquisa e extensão que tem como finalidade o desenvolvimento da ciência e tecnologia para o bem-estar social em todas as áreas da vida humana. Elas exercem um papel preponderante para o alcance desses objetivos, pois seus produtos e serviços dão a base informacional necessária para a formação profissional e acadêmica de sua comunidade.

Assim como a sociedade está em constante mutação, especialmente impulsionada pelos avanços tecnológicos das últimas décadas, as universidades e suas bibliotecas também procuram reinventar-se para acompanhar (e, se possível, promover) as transformações capazes de ampliar a qualidade de vida das pessoas em geral.

Por essas e outras tantas razões que poderiam aqui ser mencionadas, torna-se imprescindível aos bibliotecários e aos gestores de bibliotecas universitárias que eles inovem suas práticas, serviços e produtos de informação, tornando-os cada dia mais relevantes e necessários no ambiente acadêmico.

A análise das tendências aqui apresentadas a partir do estudo de caso do CRAI UPF representa um caminho para elevar a excelência do trabalho de mediação da informação e do desenvolvimento de habilidades digitais e acadêmicas fundamentais para o sucesso da formação universitária.

Por meio das inovações tecnológicas aplicadas às bibliotecas, alargam-se as fronteiras do ensino e da pesquisa em proporções quase infinitas, permitindo 
o acesso e uso de equipamentos inteligentes que criam e recriam experiências práticas ou teóricas, compartilhadas como nunca antes se tinha imaginado.

Este estudo de caso apresenta um contexto no qual são perceptíveis os esforços para uma constante implementação de serviços e produtos de base digital, e, embora avanços tecnológicos mais sofisticados ainda não sejam encontrados, é possível identificar uma aproximação com as tendências apontadas pelos relatórios NMC Horizon analisados.

O movimento contínuo de criação de novos serviços digitais será capaz de tornar as bibliotecas universitárias verdadeiros centros ativos de geração e compartilhamento de conhecimentos, cada vez mais relevantes nas áreas científicas e tecnológicas.

\section{Referências}

ANGLADA, Lluís. Bibliotecas universitarias: cabalgando la tecnología, siguiendo al usuario. El profesional de la información, [s.l.], v. 1, n. 6, p. 553$556,2012$.

BECKER, Samantha Adams et al. NMC Horizon Report: 2017 Library Edition. Austin: The New Media Consortium, 2017.

CABO RIGOL, M. El camino recorrido hacia el CRAI en la Universitat Pompeu Fabra. In: COLOQUIO INTERNACIONAL DE BIBLIOTECARIOS, 12., 2005, Guadalajara. Anais [...]. Guadalajara: Universidad de Guadalajara, 2006. p. 14153.

CASTRO FILHO, Claudio Marcondes de. O modelo europeu do Centro de Recursos para el aprendizaje y investigación (CRAI) e as bibliotecas universitárias brasileiras: convergências e divergências. 2008. Tese (Doutorado em Ciência da Informação) - Escola de Comunicação e Artes, Universidade de São Paulo, São Paulo, 2008.

CASTRO FILHO, Claudio Marcondes de; VERGUEIRO, Waldomiro. Convergências e divergências do modelo europeu do Centro de Recursos para el Aprendizaje y la Investigación (CRAI) em relação às bibliotecas universitárias brasileiras. Biblioteca Universitária, Belo Horizonte, v. 1, n. 1, p. 31-40, 2011.

CORRÊA, Elisa Cristina Delfini. Usuário, não! Interagente: proposta de um novo termo para um novo tempo. Encontros Bibli, Florianópolis, v. 19, n. 41, p. 23-40, 2014. 
EDUCAUSE. New Media Consortium (NMC). [S. l.], 2019. Disponível em: www.nmc.org. Acesso em: 20 out. 2018.

GARCÍA-QUISMONDO, Miguel Angel Marzal. La irresistible ascensión del CRAI en universidad. Ponto de Acesso, Salvador, v. 2, n. 1, p. 72-97, 2008.

GAVILÁN, César Martín. Bibliotecas universitárias: concepto y función los CRAI. [S. l.: s. $n$.], 2008. No prelo.

GUIES BBTIC. UPF. Gestió de dades de recerca: suport en la planificació i difusió. [S. l.], [2019]. Disponível em: guiesbibtic.upf.edu/data. Acesso em: 20 out. 2019.

MOREIRA, Luciana de Albuquerque. As bibliotecas universitárias de Portugal e do nordeste do Brasil: estudo sobre o impacto e mediação das tecnologias. Natal: EDUFRN, 2018.

NEVES, Jonatas Jony Furtado. A inovação nas bibliotecas universitárias brasileiras: identificando o potencial inovador. 2018. Dissertação (Mestrado em Ciência da Informação) - Programa de Pós Graduação em Ciência da Informação, Universidade Federal de Santa Catarina, Florianópolis, 2018.

SILVEIRA, Nalim Ferreira. Evolução das bibliotecas universitárias: information commons. Revista ACB, Florianópolis, v. 19, n.1, p.69-79, 2014. Disponível em: https://revista.acbsc.org.br/racb/article/view/923/0 Acesso em: 20 mar. 2019.

TAMMARO, A. M.; SALARELLI, A. A biblioteca digital. Brasília: Briquet de Lemos, 2008.

UPF. Biblioteca/CRAI del Poblenou. Barcelona. Disponível em: https://www.upf.edu/web/biblioteca-informatica/poblenou. Acesso em: 25 mar. 2019.

UPF. Biblioteca i Informàtica. Barcelona, [2019]. Disponível em: https://www.upf.edu/web/biblioteca-informatica. Acesso em: 25 mar. 2019.

ZANINELLI, Thais Batista; NOGUEIRA, Cibele Andrade; PERES, Ana Luísa Moure. Bibliotecas universitárias: uma perspectiva teórica sobre inovação em serviços informacionais. Revista Digital Biblioteconomia e Ciência da Informação, Campinas, v. 17, e019012, p. 1-20, 2019. Disponível em: http://web.archive.org/web/20200531185620/https://periodicos.sbu.unicamp.br/ ojs/index.php/rdbci/article/view/8652821 Acesso em: 10 ago. 2020.

Innovation trends in university library services: case study of the CRAI - Universitat Pompeu Fabra, Barcelona 


\begin{abstract}
This case study analyzes digital-based products and services offered by the Learning and Research Resources Center (CRAI) of the Universitat Pompeu Fabra in Barcelona, Spain. We carried out a comparative, bibliographical, and documentary research, collecting data from the New Media Consortium reports - NMC Horizon Report: 2015 Library Edition and NMC Horizon Report: 2017 Library Edition - and from the webpage of the Learning and Research Resources Center of the Universitat Pompeu FabraCRAI of the Universitat Pompeu FPF. Data were also coleted through non-participant observation carriatn that Learning and Research Resources Center many of the trends presented by the New Media Consortium reports, especially those related to the use of simpler technology. The results demonstrate the presence of many of the trends presented in New Media Consortium NMC reports, especially those using more according to the needs of the researchers and the offered allow customization contents via electronic devices such as mobile phones. A little more timidly, it is also possible to observe the customization of some services according to the needs of the researchers, as well as the offer of tools 2.0 that allow customization according to the purpose of its use as well as the delivery of contentsfound. We conclude that efforts are underway to ensure the constant implementation of digital digital-based services and products, and although more sophisticated technological advances are still to be found, it is possible to identify an approximaton by the NMC Horizon reports.
\end{abstract}

Keywords: Innovation. University libraries. CRAI. Pompeu Fabra University.

\title{
Como citar:
}

CORRÊA, Elisa Cristina Delfini; GARCÍA-QUISMODO, Miguel Ángel

Marzal. Tendências de inovação em serviços de bibliotecas universitárias: estudo de caso do CRAI - Universitat Pompeu Fabra em Barcelona, ES. Em Questão, Porto Alegre, v. 27, n. 1, p. 430-455, jan/abr. 2021. Doi: http://dx.doi.org/10.19132/1808-5245271.430-455

1 As bibliotecas digitais são entendidas como "um ambiente que reúne coleções, serviços e pessoas para apoiar todo o ciclo vital de criação, disseminação, uso e preservação de dados, informação e conhecimento" (TAMMARO; SALARELLI, 2008, p. 119). As bibliotecas híbridas são definidas como aquelas que agregam diferentes tecnologias e fontes de informação em um único ambiente, unindo o impresso ao digital num processo de transformação das bibliotecas tradicionais (TAMMARO; SALARELLI, 2008). 
Tendências de inovação em serviços de bibliotecas universitárias: estudo de caso do CRAI Universitat Pompeu Fabra em Barcelona, Espanha

Elisa Cristina Delfini Corrrêa, Miguel Ángel Marzal Garcia-Quismondo

${ }^{2}$ Termo utilizado em substituição ao termo usuário, conforme Corrêa (2014).

${ }^{3}$ BAREGHEH, A.; ROWLEY, J.; SAMBROOK, S. Towards a multidisciplinary definition of innovation. Management Decision, [s. l.], v. 47, n. 8, p. 1323-1339, 2009. Apud Neves (2018).

${ }^{4}$ Espaços físicos em formato de laboratórios comunitários usados para solução de problemas em grupo. 